\title{
Diagnostic Inferring on the Bases of Nonlinear Models
}

\author{
Z. DABROWSKI* \\ Faculty of Automotive and Construction Machinery Engineering, Warsaw University of Technology \\ Narbutta 84, 02-524 Warsaw, Poland
}

Physical phenomena accompanying destruction processes of technical systems are nonlinear and "low-energetic" by nature, while during wearing out mainly a nonlinear disturbance changes. Out of many inference techniques - on the observation basis of the state of the system - the best one is undoubtedly the well-defined mathematical model, allowing inferring 'backwards' and 'forward', which means finding the genesis and prognosis of the phenomenon. However, such model should be nonlinear. Problems related to the identification of nonlinear dynamic models in a frequency domain and a proposition of solving this problem for the needs of technical diagnostics, i.e. in situations when the observed wear out effects are significantly smaller than the dynamic effects - are discussed in the hereby paper. The bases of the proposed method constitute the discussion of possible solutions of a certain class of nonlinear differential equations and resulting from that statements on the possibility of nonlinear disturbance approximations by a series of the selected harmonic frequencies.

PACS numbers: 43.40.Ga, 45.80.+r, 46.40.Ff

\section{Introduction}

Vibroacoustic diagnostics is a relatively new field of knowledge, the development of which became possible in the last years - due to the development of measuring techniques of dynamic quantities (mainly vibrations and noise) as well as methods and means of information processing. The development of informatics forced to some extent the development of axiom of this sector.

Technical diagnostics bases on the postulate that a system destruction must lead to an energy dissipation into various parasitic processes - including mechanical vibrations and related to them noises. In the primary development period it was assumed, that the energy dissipated for vibration and noise processes is roughly proportional to the general energy dissipation. Thus, the observed vibration and noise level - in the selected frequency bands - should indicate the degree of wear or defect, it means it should be a symptom [1]. In many cases this is true. The dimensions of machine elements changes as a result of friction between them. That leads to clearances generation. An increase of clearances in kinematics pairs ${ }^{\dagger}$ increases noise and vibrations, a worn out combustion engine operates louder, etc. However, in many cases such reasoning is not sufficient. Cases of the so-called apparent "self-repairing" of structures, when an intensity of vibration-noise processes decreases for some time regardless of the worsening of failure, are quite often. The situation, when the defect development is accompanied by the form change of the observed vibrations at the same

\footnotetext{
* corresponding author; e-mail: zdabrow@simr.pw.edu.pl

$\dagger$ Kinematics pair - two movable elements of a kinematics.
}

general level, is relatively often too. A good example constitutes the evolution of spectrum of vibration accelerations of a rolling bearing with a defected bearing track, that is presented in Fig. 1.

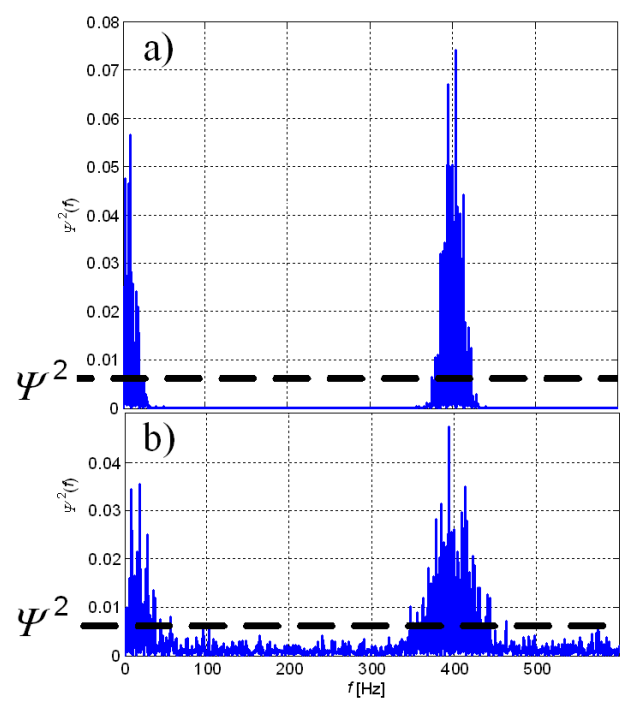

Fig. 1. Example of "iso-energy" evolution of a spectral power density of the vibration acceleration during the machine wear (rolling bearing).

Of course a modern technical diagnostics must deal with such cases, the more so as at the designing of the diagnostic monitoring systems the most important is detection of the preliminary stages of defects and observation of their evolution. This means the observation of processes in which the dissipated energy is minimal. There is only one explanation of the out-of-proportion dissipation of energy into vibration and acoustic processes of the machine defect. Since an increase of the dissipated energy 'outside' the system undergoing wearing can be 
considered to be the physical law, then the dissipation path and form must be changing.

The energy consumed for vibroacoustic processes does not have to be proportional to the total energy of parasitic processes $[2,3]$. The question arises, whether those processes can be insensitive to wearing (failure). The author, of the present paper, is of the opinion that they must be sensitive, however, it is difficult to provide a strict mathematical proof of this fact. It is only possible to refer to a wide and rich empirical material (including the one collected by the author $[1-5]$ ), which reduces to the following postulate:

Vibroacoustic processes are sensitive to degradation changes of mechanical systems even in cases when the amount of energy dissipated on the same path is not changing. In such case the form of the observed vibrations must change.

\section{Necessity of using nonlinear description}

It is assumed - for the sake of the simplification that the mechanical system undergoing degradation can be treated as a discrete set of $n$ material points. This assumption constituted an essential limitation when solely analytical models were possible to be applied. However nowadays, when the FEM type models are widely used, this assumption does not limit investigations. Discretisation is the base of calculation techniques. The performed considerations can be easily generalised into continuous models, rigid bodies, etc. The mechanical system being in a certain technical state is assumed to be describable, with a satisfactory accuracy, by a set of $n$ ordinary differential equations of the second order. In the simplest case this will be a linear system. $k$ independent excitations, related both to the machine body operations (functional) and originated from external disturbances - influence such system. Thus the system - in a linear variant is explicitly described by $3 n$ numerical parameters (inertia, torsion and damping) and $k$ functions depending evidently on time. Such system has $n^{2}$ form coefficients, and its solutions, for each generalised coordinate, is determined by the following dependency:

$$
x_{i}(t)=\sum_{i=1}^{k} p_{i}(t) h_{i}(t-\tau),
$$

where: $h_{i}=h_{i}\left(m_{n} ; c_{n} ; k_{n}\right)$ - functions of impulse response,

$m_{n}$ - inertia,

$c_{n}$ - damping coefficient,

$k$ - elasticity.

Those solutions can be easily reduced into the frequency domain by means of the Fourier transform. Then the complex dependences are obtained:

$$
X_{i}(\omega)=\sum_{i=1}^{k} P_{i}(\omega, i) H_{i}(\omega, i),
$$

where: $X_{i}, P_{i}$ - Fourier transform of coordinates and excitations - respectively, while $H_{i}$ - spectral transmittances, or — omitting phase relations:

$$
G_{x x}=\sum P_{x x}|H|_{i}^{2},
$$

where: $G_{x x}, P_{x x}$ - spectral concentrations of input and output signals — respectively, while $|H|_{i}^{2}$ — coefficients of power density amplification.

The system under consideration changes the technical state, what expresses itself by changes of an arbitrary number of coefficients $c_{i}, k_{i}$ and (less frequently) $m_{i}$. This change changes, of course, all functions of impulse responses as well as transmittances and coefficients of amplification, but does not change a spectral power density of excitations. Power spectrum of solutions will change, however, the zero zones will be the same. Such reasoning illustrates the fact that changes of coefficients of the linear set of differential equations can not generate - at the same excitation - the answer in the other frequency range (although amplitudes can arbitrarily change).

Thus, the only possibility of obtaining solution transformation from the form "a" to "b", presented in Fig. 1, is the change of the linear system to nonlinear one or the change of the nonlinear parts of characteristics of the system, which was nonlinear from the very beginning.

\section{3. "Diagnostic" model}

There are many ways and techniques of building diagnostic tests and monitoring systems corresponding to them. The coherent and verified theory of diagnostic experiments was developed $[1,6]$. Various "intelligent data bases" are the big success in recent years. "Self-learning" systems based on the neural networks theory enabled to solve several tasks - previously insoluble.

However, the best way to establish a prognosis is to have the properly identified mathematical model of the failure growing. Such model should be nonlinear, as results from the above given considerations. Additionally, it should be taken into account that the observed vibroacoustic effects, which accompany the defect propagation, are very small as compared to the dynamic effects of the machine operation. Thus, the observation concerns small changes of parasitic processes of energy - sometimes by several orders of magnitude - different from "functional" dynamic processes. Building the precise dynamic machine model which would be sensitive to all initial stages of defects is practically unrealisable. Additional difficulty constitutes the fact that the system degradation is a random process. The result of the observation is also a random one. Even if it is assumed, that the mechanical system operation is describable, with a good approximation, in a deterministic way the relation: observed signal $\Leftrightarrow$ mathematical model will be reduced to the form:

$$
S_{t}\{y(t, n, r, \theta)\} \Leftrightarrow F\left(p_{i}, h_{i}\right),
$$

where $\{y(:,:)\}$ denotes the observed vibration or noise signal, which in its simplest form is the random process of four following variables:

$t$ - observation time,

$n$ - discriminant of the machine under testing, 
$r$ - coordinate determining location of the measurement sensor,

$\theta$ - exploitation time (life time) or other discriminant of the machine state.

The right member of the equation denotes the modelling result. Since the system has to be nonlinear, then instead of the Eq. (4) there is a combined function of excitations and impulse responses. In order to make this relation possible it is necessary to introduce the selection operator and averaging $S_{t}$. This, on the one hand, enables the selection of interesting parameters from the signal that is being observed and, on the other hand, enables the comparison of both members by substituting the random process by its determined characteristics. The relevant metric should be also determined, transfer functions subjected to state parameters and condition solved in consideration of these parameters. Obviously the solution requires the same number of equations (measurement points) as the number of parameters being introduced. The model constructed in such a way can be called the diagnostic model. In order to be able to operate this model must be properly identified, what due to the ambiguity of the so-called inverse identification task is extremely difficult. In addition, the analysis limited to time domain (nonlinear system does not undergo Fourier transforms) is extremely difficult in the diagnostic task. This situation prompted the author of the present paper to formulate the proposition which is described in the next paragraph.

\section{Proposition of solution of the building nonlinear diagnostic model}

The needs of the vibroacoustic diagnostics require to have the model describing the signal propagation path from the place of the expected defects to the observation point. Thus, the selected part of the machine system and not the entire system can be described in details. Inputs and outputs from the object under modelling should be indicated from the observation (measurement) [7].

The following assumptions should be adopted:

- The object able for operations is described, with a good accuracy, by a set of ordinary linear differential equations of the second order,

- Degradation of the system introduce a nonlinear disturbance, which is changing during further wear (failure).

The linear system can be easily reduced to the main coordinates in which equations are uncoupled:

$$
\ddot{\xi}_{i}+\omega_{0 i}^{2} \xi=p_{i}(t)
$$

where $\omega_{0 i}$ - the ith natural frequency.

Left members of equations will be uncoupled while the right ones will remain the complicated functions of the remaining coordinates:

$$
\ddot{\xi}_{i}+\omega_{0 i}^{2} \xi=\Phi_{k}\left(\dot{\xi}_{1}, \ldots, \dot{\xi}_{n} ; \xi_{1}, \ldots, \xi_{n} ; t\right)
$$

Such system can be solved only in an approximate way. There is only one possibility of predicting the form of the solution on the basis of the generalised approximate analytic solution for small number of the degrees of freedom $[1,8]$. In the first approximation the following form is obtained ${ }^{\ddagger}$ :

$$
\begin{aligned}
\xi_{j} & =a_{j}(t) \cos \psi_{j}(t) \\
& +\frac{1}{(2 \pi)^{1+n}} \sum_{n} \sum_{m} \frac{\operatorname{exp~} \mathrm{i}\left(m \Omega+\sum_{k} \psi_{k} n_{k}\right)}{\omega_{o k}^{2}-\left(m \Omega^{2}+\sum_{k} n_{k} \omega_{o k}^{2}\right)} \\
& \times \underbrace{\int_{0}^{2 \pi} \int_{0}^{2 \pi} \int_{0}^{2 \pi} \cdots \int_{0}^{2 \pi} f_{k}}_{n+1} \\
& \times \exp _{m, n, k}\left\{(-\mathrm{i})\left[m \Omega t+\sum_{k} n_{k} \psi_{k}\right]\right\} \mathrm{d} \psi_{1} \ldots \mathrm{d} \psi_{n} \mathrm{~d} t \\
& \left\{N-\left(m ; n ; k: \omega_{o k}^{2}-\left(m \Omega^{2}+\sum_{k} n_{k} \omega_{o k}^{2}\right)=0\right\},\right.
\end{aligned}
$$

where $f_{k}$ is as follows:

$$
\begin{aligned}
& f_{k}=\Phi_{k}\left(a_{1} \cos \psi_{1}, \ldots, a_{k} \cos \psi_{k} ;-a_{1} \omega_{o 1} \sin \psi_{1}, \ldots,\right. \\
& \left.\quad-a_{k} \omega_{o k} \sin \psi_{k} ; \Omega t\right)
\end{aligned}
$$

For the sake of readability it was assumed, that all excitations are polyharmonic of the commensurable frequencies and the common measure of these frequencies were denoted by $\Omega^{\S}$.

When vibrations of a certain harmonic group do not occur in the observed spectrum, one is permitted to state the zeroing criteria of coefficients of these components. These criteria identify coefficients of power series expansion of nonlinear characteristics of elasticity and damping in a relatively simple way, according to:

$$
\begin{aligned}
& A_{m_{o}, n_{o}, k_{o}}=0 \Leftrightarrow \underbrace{\int_{0}^{2 \pi} \int_{0}^{2 \pi} \ldots \int_{0}^{2 \pi}}_{n+1} f \\
& \quad \times \exp \left(-\mathrm{i}\left(m_{o} \Omega+\sum n_{k_{o}} \psi_{k_{o}}\right)\right) \\
& \quad \mathrm{d} \psi_{1} \ldots \mathrm{d} \psi_{n} \mathrm{~d} t_{n+1}=0 \\
& \Rightarrow K\left(k_{1}, \ldots, k_{n} ; m_{1}, \ldots, m_{n} ; c_{1}, \ldots, c_{n}\right)=0 .
\end{aligned}
$$

However, regardless of the possibility of finding depen-

\footnotetext{
$\ddagger$ The autor applied here the KB method, however the identical form can be obtained when applying e.g. variance methods (Ritz'es, Galerkin's) [6].

$\S$ An assumption of an arbitrary number of incommensurable frequencies does not change the solution form but only multiplication factor of integrations.
} 
dencies between system parameters on the basis of the occurrence or not occurrence of components of system responses of various frequencies, this is not the explicit inverse identification method of elasticity and damping. The solution (7) is only pertinent far from the resonance zones. The condition (9) is only the necessary condition of the spectrum range of the solution and does not give any information concerning the amplitudes (!).

However, the form of the solution (7) is an additive one. An influence of a nonlinear disturbance is written in the form of a series of successive corrections (each successive correction has the same form and corrects only functions $f_{k}$ ). Thus, the dependence: signal $\Leftrightarrow$ model for the systems with the nonlinear disturbance can be written:

$$
S_{t}\{y(t, n, r, \theta)\}=\sum p_{i}(t) h_{i}(t-\tau)+\varphi+\psi,
$$

where $\varphi$ denotes the vector of correction functions, which brings the linear system solution into the nonlinear one, $\psi$ - measurement noise.

Since functions $\varphi$ are here functions of a single variable the equation can be brought into a frequency domain by applying the Fourier transform:

$$
S_{\omega} F S_{t}\{y(t, n, r, \theta)\}=\sum P_{i} H_{i}+\Phi+\Psi,
$$

where Fourier transforms of values from Eq. (10) are marked by capital letters, and $S_{\omega}$ denotes the selection operator in the frequency domain (filtration operator).

The sought after form of solution enables to identify the diagnostic model, according to the following procedure: $z_{i}$ denotes parameters of the linear model at the "zero" $\left(\theta=\theta_{0}\right)$ state, which are difficult to the direct determination. The simple condition of the parametric identification of the linear system is built on the basis of Eq. (11):

$$
\begin{gathered}
\left|S_{\omega} F S_{t}\left\{y\left(t, n, r, \theta_{0}\right)\right\}-\sum_{\Downarrow} P_{i} H_{i}\left(z_{1}, \ldots, z_{n}, \ldots\right)\right|<\delta, \\
z_{i}
\end{gathered}
$$

where $\delta$ denotes the permissible identification error, when $\delta>\psi$.

For the other selected state $\theta_{k}$ :

$$
\begin{gathered}
\left|S_{\omega} F S_{t}\left\{y\left(t, n, r, \theta_{k}\right)\right\}-\sum_{i} P_{i} H_{i}\left(z_{1}, \ldots, z_{n}, \ldots\right)+\Psi_{k}\right| . \\
<\delta \\
\Downarrow \\
\Phi_{k}(\theta, \ldots)=F^{-1} \Phi
\end{gathered}
$$

In order to make the model diagnostically useful one other empirical verification has to be done for $\theta_{n} \neq \theta_{k}$. In case of large discrepancies functions $\theta_{k}$ should be corrected. Such procedure leads, generally, to positive results.

\section{Conclusions}

The presented hereby proposition of considering the evolution of the mechanical systems as the increase of the nonlinear disturbance together with its two stage identification process (where in the first stage the qualitative analysis is performed and in the second stage the quantitative determination of correction functions is done), reduced itself to several detailed applications $[3,7,8,10]$.

Finally the diagnostic task, at such way of modelling, reduces to the following formalism:

$$
\begin{gathered}
Y\left(\omega, \Theta_{m}\right)=\sum P_{k}(\omega) H_{k}\left(q_{i}, z_{i}\right)+\Phi^{*}\left(\Theta_{m}\right)+\psi \\
Y\left(\omega, \Theta_{n}\right)=\sum P_{k}(\omega) H_{k}\left(q_{i}, z_{i}\right)+\Phi^{*}\left(q_{i}, \Theta_{n}\right)+\psi \\
\Downarrow \\
\Delta_{m n} Y(\omega)=-\Phi^{*}\left(\Theta_{m}\right)+\Phi^{*}\left(q_{i}, \Theta_{n}\right)+\psi^{*} \\
=\Delta \Phi^{*}\left(q_{i}, \Theta_{m}, \Theta_{n}, \psi^{*}\right) \\
\Downarrow \\
q_{i}=\Delta \Phi^{*)-1} \Delta_{m n} X\left(\Theta_{m}, \Theta_{n}, \psi^{*}\right),
\end{gathered}
$$

where $q_{i}$ is the sought after "initial" defect parameter and $\psi g$ - the resultant of the measurement noise disturbation.

The problem constitutes finding the inverse transform $\Delta \Phi^{*(-1)}$, what in practice means the proper selection of the metric (measure) differentiating the vibration (noise) spectrum of the system able for operations and the defected one. In the author's opinion the coherence methods $[9,10]$ or the proposed by Batko reduction of the task into the stability analysis of phase trajectories [11] — are functioning well.

\section{References}

[1] Z. Dąbrowski, Machine Dynamic Problems, Vol. 4, (1992).

[2] W. Batko, Z. Dąbrowski, J. Kiciński, Nonlinear effects in technical diagnostics, Publishing and Printing House of the Institute for Sustainable Technologies NRI, Warsaw 2008, p. 303.

[3] I. Komorska, Study on the effect of assembly errors on the vibration of serial transfer power systems, Ph.D. Thesis, Warsaw University of Technology, Warsaw 1999 (in Polish).

[4] J. Pankiewicz, Use of non-linear effects in diagnosing of viscoelastic elements, Ph.D. Thesis, Warsaw University of Technology, Warsaw 2002 (in Polish).

[5] Diagnostic Engeneering, Eds. B. Żółtowski, C. Cempel, Publishing and Printing House of the Instytute for Sustainable Technologies - NRI, Radom 2004, p. 1308 (in Polish).

[6] Z. Osiński, Dumping of vibration, Polish Scientific Publishers PWN, Warsaw 1997 (in Polish).

[7] Z. Engel, Environmental protection from vibrations and noise, Polish Scientific Publishers PWN, Warsaw 2001 (in Polish).

[8] J.A. Mitropolskij, G.P. Homa, Matemacziskoje obosnowanije asimptoticzeskich metodow nieliniejnoj mechaniki, Naukowaja Dumka, Kijev 1983 (in Russian).

[9] Z. Dąbrowski, in: Modeling and identification of nonlinear system for technical diagnostics, The 8th International Congress on Sound and Vibration, Hong Kong (China) 2001 p. 1083.

[10] J. Dziurdź, W.W. Skórski, Diagnostyka 1, 63 (2009).

[11] W. Batko, Problems of Machines Operation and Maintenance 3 (151), 147 (2007). 